$5^{\text {th }}$ International Conference on Industrial Engineering and Industrial Management

XV Congreso de Ingeniería de Organización

Cartagena, 7 a 9 de septiembre de 2011

\title{
Heurística de asignación en tiempo real de vehículos a tareas de acarreo intermodal
}

\author{
Heuristic of real-time assignment of intermodal drayage task
}

\author{
Alejandro Escudero, Jesús Muñuzuri, José Guadix y Carlos Arango \\ Grupo de Ingeniería de Organización. Dpto. de Organización Industrial y Gestión de Empresas. \\ Escuela Técnica Superior de Ingenieros de Sevilla. Universidad de Sevilla. \\ Camino de los Descubrimientos s/n, 4l 092 Sevilla. \\ aescudero@esi.us.es, munuzuri@esi.us.es, guadix@esi.us.es, cap@esi.us.es
}

\begin{abstract}
Fecha de recepción: 7-9-2011
\end{abstract}
Fecha de aceptación: 19-9-2011

Resumen: La asignación de tareas en sistemas de transporte ha sido tradicionalmente hecho una vez al día. Al comienzo de la jornada, el decisor establece que tareas deben ser llevadas a cabo por cada vehículo. Entonces, atascos, averías y cualquier problema inesperado causaría grandes retrasos e inconvenientes sobre lo planificado. En este trabajo se propone el uso de información en tiempo real relativa a la posición de los vehículos para solventar el problema. De este modo, el decisor está permanentemente reconsiderando mejoras en la asignación si las condiciones lo aconsejan. Un mecanismo heurístico ha sido elegido para solventar el problema dada la necesidad de una gran velocidad computacional.

Palabras clave: transporte intermodal, acarreo terrestre, heurísticas.

Abstract: The scheduling of transportation systems has traditionally been done once a day. At beginning of a working day, the planner establishes which tasks will be carried out by each vehicle. Then, traffic jam, breakdown and any unexpected problem will cause delays on our timetable. In this paper, we propose to use real-time vehicle position knowledge to solve this problem. So, the planner is permanently enabled to reallocate tasks as the problem conditions change. As both drayage problem is a NP-Hard problem and a high-speed procedure is required, exact methods are not computationally feasible. So, a heuristic algorithm has been implemented to perform the problem described.

Keywords: intermodal transport, drayage, heuristic.

\section{Introducción}

El transporte por carretera es el principal modo de envío de mercancías en el ámbito terrestre. Observando los últimos datos proporcionados por el Instituto Nacional de Estadística, el transporte interregional de mercancías en el año 2008 fue de I 19.559 $\mathrm{Tn} \cdot \mathrm{Km}$ en el caso del transporte por carretera y de tan sólo $10.287 \mathrm{Tn}$. Km en el caso del transporte ferroviario.Esta contundente preferencia del mercado hacia la carretera se debe principalmente a una serie de ventajas que esta presenta para el movimiento de bienes dentro de España; entre ellas cabe destacar una gran flexibilidad de operación, la posibilidad de comenzar un envío desde cualquier lugar y en cualquier momento.
Sin embargo, el aumento de la congestión en las principales vías españolas y la creciente preocupación por la sostenibilidad han hecho a los diferentes gobiernos buscar modos alternativos de transporte.

Desde las diferentes administraciones, se está señalando al transporte intermodal como una alternativa. La intermodalidad, definida por la Comisión Europea como una característica de un sistema de transportes en virtud de la cual se utilizan de forma integrada al menos dos modos de transporte diferentes para completar una cadena de transporte puerta a puerta, permite mediante un planteamiento global una utilización más racional de la capacidad de transporte disponible. La intermodalidad no pretende imponer una opción modal, pero si permite 
utilizar de una forma más adecuada el ferrocarril, las vías navegables y el transporte marítimo, que por sí solos no permiten el transporte puerta a puerta. La intermodalidad, por tanto, hace uso de transportes que presentan una gran economía de escala (ferrocarril, marítimo,...) para llevar a cabo el mayor tramo posible del desplazamiento, siendo el transporte por carretera usado para llevar a cabo los trayectos finales o entre terminales.

Para que la intermodalidad sea una realidad en trayectos inferior a los $700 \mathrm{~km}$ se hace necesaria una reducción de sus costes (Escudero et al., 2007). Casi el $40 \%$ de los costes de dicho modo de transporte se encuentran localizados en los trayectos finales (Morlok y Spasovic, 1994), por tanto una adecuada gestión de los mismos podría hacer de la intermodalidad un modo mucho más competitivo. Este transporte entre terminales y clientes finales es Ilamado acarreo o drayage.

En el modelo planteado resuelve el problema del acarreo en un entorno donde los tiempos de transito de nuestros viajes no son deterministas, sino que presentan una cierta aleatoriedad. Para resolver el inconveniente del desconocimiento a priori de la duración de los viajes, se hace uso de la información que las nuevas tecnologías pueden proporcionar, gracias a las cuales se tiene conocimiento exacto de la posición de la flota y comunicación permanente con todos los conductores. De este modo, se podrán modificar la planificación diaria dependiendo de las circunstancias que se vayan produciendo a lo largo del día.

\section{Arquitectura propuesta}

La metodología propuesta para una asignación en tiempo real de vehículos a tareas de acarreo se basa principalmente en dos suposiciones. Por un lado se debe de disponer de una tecnología que permita conocer en cada instante donde se encuentran localizados los vehículos de nuestra flota y por otro lado debe de existir la posibilidad de comunicación inmediata con dichos vehículos. Ambas suposiciones pueden ser consideradas como ciertas; para la localización de la flota, gracias a los sistemas de posicionamiento vía satélite, como el americano GPS o el europeo GALILEO, o a los sistemas de posicionamiento por triangulación; y para la comunicación, gracias a las comunicaciones móviles como GSM, GPRS, UMTS, o incluso TETRA.

El algoritmo que se ha desarrollado formaría parte de un sistema global de planificación que se llamará: Sis- tema GALILEO-Drayage. Este sistema recibiría diversos tipos de informaciones, que serían posteriormente utilizadas como datos para la ejecución del algoritmo:

- Solicitudes de tareas, que serían incorporadas directamente al sistema por parte de los clientes del mismo (cargadores y receptores de mercancía).

- Información sobre la posición de los vehículos en el viario, a través del sistema Galileo.

- Información sobre la compleción de tareas, proporcionada por los conductores de los vehículos al sistema a través del correspondiente interfaz de comunicaciones.

- Por último, y de manera opcional, el sistema podría contar con información actualizada sobre el estado del viario (fundamentalmente el grado de congestión y la velocidad media en los diferentes tramos del mismo), en caso de que esta información estuviera disponible.

La Figura I muestra, de modo esquemático, la arquitectura prevista del sistema. La comunicación entre las interfaces y el sistema, o entre los clientes y el sistema, se realizaría a través de internet, lo que hace necesaria la existencia de interfaces de comunicaciones con los vehículos. Además, queda reflejada la ubicación del algoritmo de optimización dentro de la arquitectura global, como el encargado de asignar tareas pendientes a vehículos en tiempo real. Esta arquitectura permite al algoritmo de optimización realizar una asignación más adecuada de las tareas, contemplando las incidencias que hayan podido suceder en la realización de las tareas llevadas a cabo o en nuestros vehículos. De esta manera la asignación encontrada al principio del día podrá ir cambiando si las circunstancias lo requieren.

La posibilidad de poseer información en tiempo real sobre el viario permitiría no sólo reorganizar las rutas atendiendo a incidencias en nuestros vehículos y en el tiempo de ejecución de las tareas previas, sino realizar una asignación más fina atendiendo a las circunstancias de tráfico en cada momento.

\section{Definición del problema}

El problema del drayage (DP) puede ser definido como un VRPTW (Vehicle routing problem with time windows) con carga completa. Se trata de un problema donde los vehículos o bien están totalmente cargado si llevan un contenedor, o bien están vacíos 
Figura I

Arquitectura del sistema GALILEO-Drayage

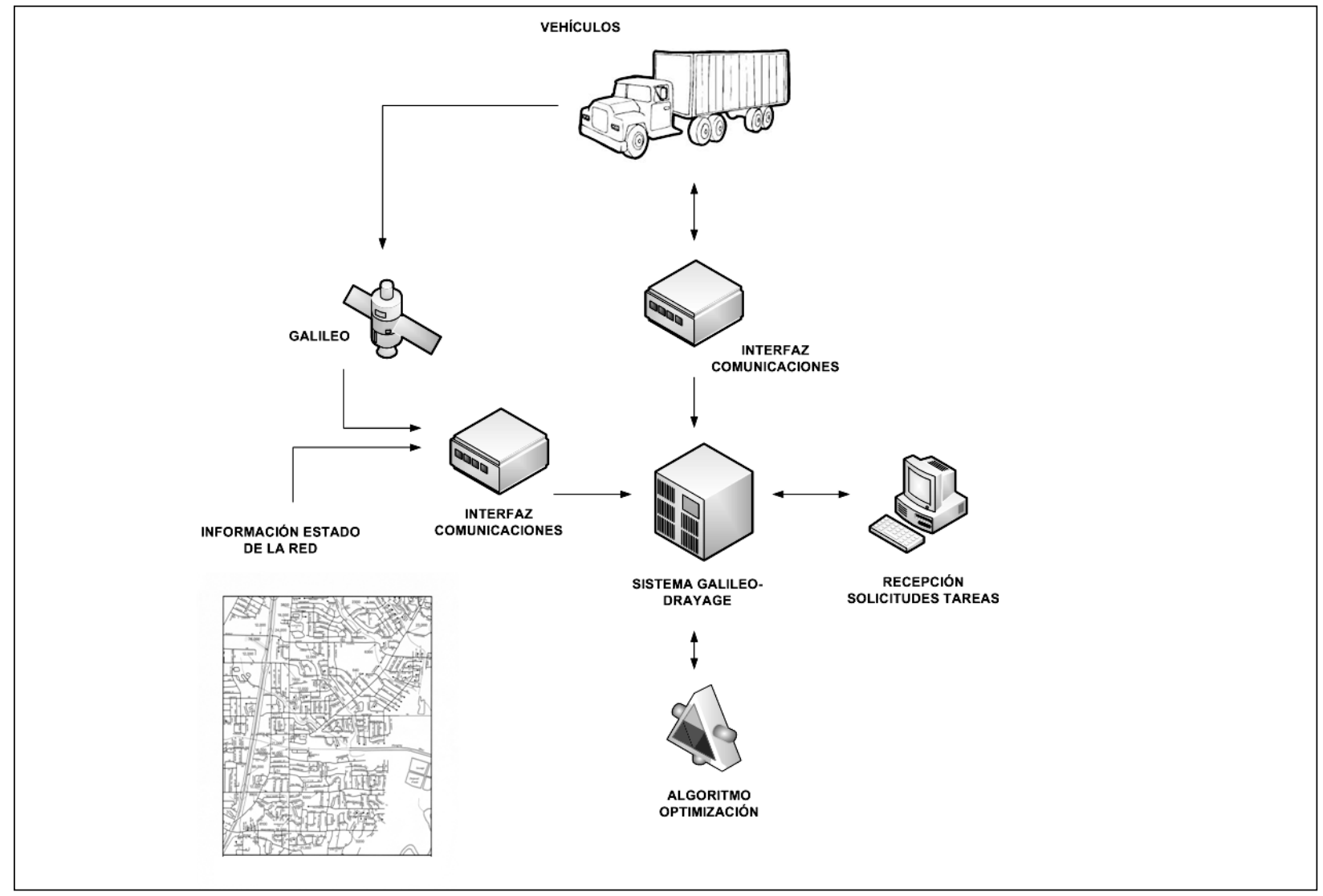

en caso contrario. Además, por simplificar, se asumirá que los contenedores son homogéneos, todos del mismo tipo y tamaño.

El problema consistirá en encontrar la mejor asignación posible de tareas a vehículos con el objetivo de minimizar los costes totales. Estos costes incluirán costes fijos por vehículo usado, costes por distancia recorrida, y al encontrarnos en entornos con tiempo de transito estocástico, costes por llegada temprana o tardía al origen o al destino.

Las tareas podrán ser de dos tipos, o tareas de recogidas de contenedores en la terminal, o tareas de entrega de contenedores en la terminal. Cada tarea $t \in$ $\mathrm{T}$, además de un origen y un destino, tendrá asociada una ventana temporal que limita el tiempo en el que estas pueden ser realizadas. Las ventanas podrán ser definidas de dos maneras: por el inicio de la tarea, $\left[a_{i n i}^{t}, b_{i n i}^{t}\right]$, o por la finalización de la misma, [ $\left.a_{\text {fin }}^{t}, b_{\text {fin }}^{t}\right]$. Que la ventana esté referida al inicio de la tarea o a la finalización de la misma dependerá del la tarea en cuestión a realizar. Si se necesita recoger un contenedor de la terminal la ventana estará definida al inicio de la tarea, por el contrario si la tarea consistie- ra en la entrega del contenedor en la terminal, la ventana estaría fijada al final de la tarea. La penalización de llegar a una posición determinada fuera de la ventana temporal dependerá también de la tarea en cuestión, si se trata de la recogida de un contenedor de la terminal para llevarlo a su destino final, el llegar tarde supondrá una penalización por almacenaje que se supondrá proporcional al tiempo de espera, $c_{w}$. Sin embargo, si se trata de la entrega de un contenedor en terminal para que este sea enviado por tren hacia otra terminal, la llegada tardía podría suponer la perdida del servicio y la espera en la terminal hasta una siguiente salida, por lo que el coste podría ser mucho más elevado y no sería proporcional a tiempo de retraso, c. La Figura 2 superior hace referencia a una tarea de recogida de mercancías de la terminal, mientras que la figura inferior hace referencia a una tarea de entrega de mercancías.

\section{Heurística de inserción, mejora y reparto}

Debido al carácter dinámico en la asignación de las tareas, y la posibilidad de que las condiciones del via- 
Figura 2

Ventanas temporales

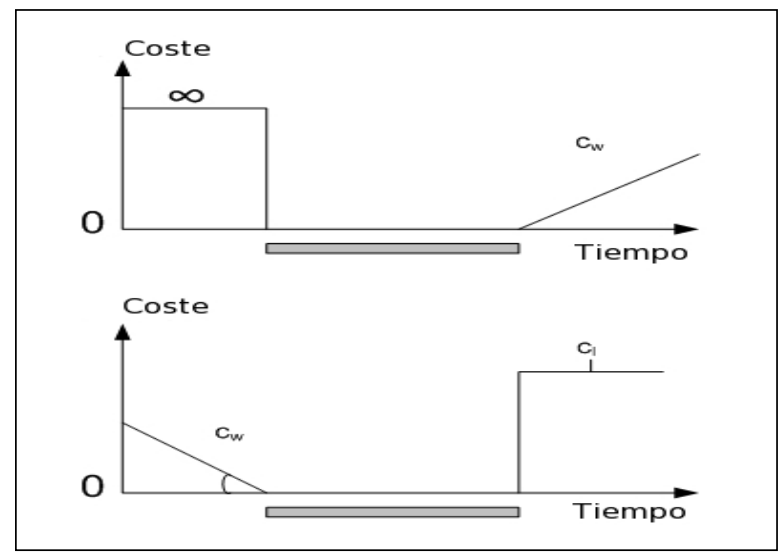

rio vayan cambiando a lo largo del día. Es necesario que el algoritmo de asignación de vehículos a tareas sea lo suficientemente veloz. El algoritmo propuesto es una modificación del algoritmo de Caris y Janssens (2009). Al poder realizarse reoptimizaciones a lo largo de todo el horizonte de planificación se ha añadido la posibilidad de que los vehículos estén en cualquier posición. Además, Caris y Janssens (2009) en su etapa de mejora, siempre intenta reducir el número de vehículos, esto lo consigue cargando de tareas a los vehículos mas baratos. El algoritmo aquí mostrado intenta reducir el número de vehículos nuevos a usar de esa misma forma, pero sin embargo reparte lo más uniformemente posible las tareas entre los vehículos que ya han sido usados.

Como se ha comentado con anterioridad, los vehículos pueden encontrarse en cualquier situación, además este puede estar o bien ocupado, o bien libre, o bien asignado a una tarea que todavía no ha comenzado a realizar. Con el objeto de salvar la problemática que toda esta información conllevaría en un entorno dinámico, se ha considerado apropiado introducir toda esta información en unas tareas «ficticias»». De esta manera, el estado de los vehículos será tratado como una tarea más, con la condición de que tienen que ser las primeras en servirse. La información que contienen dichas tareas es simple, y dependerá del estado del vehículo:

- Vehículos libres y asignados, el origen y destino será la posición actual, y su ventana temporal será asignada al origen y tendrá como limites temporales el momento actual. De esta forma esta tarea «ficticia» se hará inmediatamente.

- Vehículos ocupados, el origen será la posición actual, mientras que el destino será el destino de la tarea que se está llevando a cabo. La ventana temporal en origen tendrá como limites temporales el momento actual, para forzar que la «tarea» sea inmediatamente llevada a cabo (obligar que el vehículo siga realizando la tarea), y la ventana temporal en destino será la ventana en destino de la tarea que el vehículo está realizando, en el caso de que esta exista.

La heurística propuesta se divide en 2 partes, una heurística de inserción que construirá una solución inicial a partir de la unión de pares de tareas que supongan un ahorro, y una etapa de mejora que incluirá 3 búsquedas locales. La heurística de inserción consta de dos fases: una primera fase de emparejamiento de tareas y una segunda de la inserción de las mismas en diferentes rutas.

La primera fase consiste en el emparejamiento de tareas de entrega $(D)$ y de recogida $(P)$ de contenedores, de esta forma se ahorrará recorridos innecesarios con la terminal $(T)$, en lugar de realizar la secuencia de movimientos: Posición Inicial - D -T $P-T$, se llevará a cabo: Posición Inicial - D - P - T. Este ahorro se muestra claramente en la Figura 3.

Será necesario tener en cuenta que no todos los emparejamientos podrán ser llevados a cabo por cuestiones temporales y/o espaciales. Para cada enlace posible se evaluará el tiempo mínimo de espera y el ahorro que supone llevarlo a cabo. Atendiendo a estos dos valores, se ponderará la conveniencia de cada enlace, eligiendo los que sean más favorables y no presenten tareas repetidas.

La segunda fase del algoritmo de inserción consistirá en la construcción de las rutas, los emparejamientos anteriores junto con las tareas que no han podido ser emparejadas se ordenan en creciente orden según su último tiempo de comienzo posible, y se van insertando en las rutas de los distintos vehículos, comenzando por vehículos de menor coste.

Una vez que se ha construido la ruta inicial, esta será mejorada a través de una búsqueda local en con tres vecindades. La primera se vecindad se trata de cruzar parejas. Dos pares de P-D son seleccionados (i,j) y $(g, h)$, se realiza el cruce $(i, h)$ y $(g, j)$, y se examina si es posible realizar el cruce e insertar después los pares en las rutas. De todos los posibles cruces se elige el que suponga mayor ahorro. La segunda vecindad consiste en la combinación de rutas, de esta forma se podría ahorrar en el uso de vehículos. La tercera vecindad intentará insertar pares de los vehículos con mayor coste en los vehículos más baratos. 
Figura 3

Emparejamiento de tareas

(c) (c2) (c3)

\section{Re-optimización}

Como se ha comentado con anterioridad, la heurística propuesta posibilita la re-optimización a lo largo de la jornada laboral, de forma que dependiendo de las circunstancias reales de cada momento la planificación de la jornada pudiera ir cambiando y de ese modo ahorrar costes y esperas innecesarias. Los pasos a seguir cada vez que una re-optimización es llevada a cabo se muestran a continuación:

\section{Re-optimización}

I. Esperar a que ocurra un evento de re-optimización

2. Leer la posición de los vehículos.

3. Chequear el estado de las tareas (Pendientes, En Proceso, Realizadas) y de los vehículos (Libres, Ocupados, Asignados)

4. Actualizar las tareas ficticias con la información de 2. y 3.

5. Re-optimización (Algoritmo de Inserción).

6. Actualizar todas las variables sobre el estado de los vehículos.

7. Ir a I.

Como evento de re-optimización se han considerado dos posibilidades. En la primera la re-optimización se lanza cada vez que un vehículo termina una tarea, en la segunda el lanzamiento de la misma será cada un cierto tiempo fijo, en nuestro caso cada 15 minutos.

\section{Resultados}

Un resumen de los resultados alcanzados es mostrado en las siguientes tablas. El procedimiento fue testado en 4 clases de problemas con 10 instancias de cada uno de ellos. Estas 40 instancias resultantes se testaron en 100 entornos de velocidades diferentes. Todas las instancias tenían 30 tareas a realizar. El procedimiento fue comparado con el procedimiento clásico de realizar una planificación «ciega» al principio del día, tomando como datos los tiempos de transito esperado. Se utilizó el propio algoritmo de Caris y Janssens (2009) como punto de referencia.

La Tabla I muestra tanto la mejora en costes media obtenida, como la mejora máxima, tanto en el caso de realizar la re-optimización cada un cierto tiempo fijo, o cuando una tarea es terminada. La Tabla 2 muestra un promedio de las tareas que son servidas con retraso.

\section{Conclusiones}

A través de la adaptación de un algoritmo estático a un problema de asignación dinámica y su aplicación 
Tabla I

Resultados: Media de mejora en Coste

\begin{tabular}{|c|c|c|c|c|c|c|}
\hline \multirow{2}{*}{$\begin{array}{c}\text { Clase } \\
\text { de problema }\end{array}$} & \multirow{2}{*}{$\begin{array}{c}\text { Tamaño } \\
\text { del Hinterland }\end{array}$} & \multirow{2}{*}{$\begin{array}{c}\text { Ancho } \\
\text { de las ventanas } \\
\text { temporales }\end{array}$} & $\begin{array}{c}\text { Mejora } \\
\text { media (\%) }\end{array}$ & $\begin{array}{c}\text { Máxima } \\
\text { mejora (\%) }\end{array}$ & $\begin{array}{c}\text { Mejora } \\
\text { media (\%) }\end{array}$ & $\begin{array}{c}\text { Máxima } \\
\text { mejora } \\
(\%)\end{array}$ \\
\hline & & & $\begin{array}{c}\text { Finalizado } \\
\text { de tareas }\end{array}$ & $\begin{array}{c}\text { Finalizado } \\
\text { de tareas }\end{array}$ & $\begin{array}{l}\text { Cada } \\
15 \mathrm{~min} .\end{array}$ & $\begin{array}{l}\text { Cada } \\
15 \mathrm{~min} .\end{array}$ \\
\hline 1 & 50 & $60-120$ & 9.66 & 33.85 & 12.17 & 37.98 \\
\hline 2 & 50 & $90-240$ & 5.38 & 35.19 & 6.95 & 35.82 \\
\hline 3 & 100 & $60-120$ & 3.91 & 33.23 & 5.05 & 35.80 \\
\hline 4 & 100 & $90-240$ & 4.25 & 35.19 & 4.69 & 35.82 \\
\hline
\end{tabular}

Tabla 2

Resultados: Promedio de tareas realizadas con retraso

\begin{tabular}{|c|c|c|c|c|c|c|}
\hline \multirow{2}{*}{$\begin{array}{c}\text { Clase } \\
\text { de problema }\end{array}$} & \multicolumn{5}{|c|}{ Retrasos en origen } & \multicolumn{3}{c|}{ Retrasos en destino } \\
\cline { 2 - 7 } & Sin & $\begin{array}{c}\text { Finalizado de Re-optimización } \\
\text { de tareas }\end{array}$ & Cada I 5 min. & Sin & $\begin{array}{c}\text { Finalizado } \\
\text { de tareas }\end{array}$ & Cada I5 min. \\
\cline { 2 - 7 } & 2,52 & 0,15 & 0 & 2,64 & 0,92 & 0,63 \\
\hline 1 & 1,46 & 0,04 & 0 & 1,88 & 0,73 & 0,63 \\
\hline 2 & 2,23 & 0,25 & 0,09 & 2,64 & 1,01 & 0,87 \\
\hline 4 & 1,69 & 0,17 & 0,04 & 2,43 & 0,89 & 0,74 \\
\hline
\end{tabular}

a una serie de instancias, se ha demostrado los beneficios de incorporar el conocimiento en tiempo real de la posición de los vehículos. En más del 95\% de los casos mejoramos al planteamiento clásico de realizar una sola planificación al principio de la jornada. Nuestros resultandos muestran una mejora media entre el 5 -10\% y una mejora en los retrasos en entrega.

Para poder llevar a cabo esta metodología de asignación dinámica es necesario un algoritmo lo suficientemente veloz que nos de resultados en tiempos inferiores a un minuto, es por eso que se ha optado por este procedimiento heurístico.

\section{Agradecimientos}

Esta investigación fue financiada por el programa EraStar Regions y la Consejería de Innovación y Ciencia de Andalucía, SR-0 197/2008 (Proyecto Galileo-Drayage).

\section{Bibliografía}

CARIS, A. y JANSSENS, G.K. (2009), A local search heuristic for the pre- and end- haulage of intermodal container terminals. Computers \& Operations Research, vol. 36, pp 2763-2772.

ESCUDERO,A.; DELGADO, M.C.; MUÑUZURI, J.y ONIEVA, L., «Modelo de Ayuda a la Decisión en Transporte Intermodal». En: Actas International Conference on Industrial Engineering and Industrial Management. Madrid. 2007. Pag. 399-400.

MORLOK, E.y SPASOVIC, L. ( 1 994), Redesigning rail-truck intermodal drayage operations for enhanced service and cost performance. Journal of the Transportation Research Forum, vol. 34, no. I, pp. 16-31. 